\title{
Simulations of dense agitated solid-liquid suspensions - effects of the distribution of particle sizes
}

\author{
J.J. Derksen \\ School of Engineering, University of Aberdeen, Aberdeen, UK \\ jderksen@abdn.ac.uk \\ Submitted to Chemical Engineering Science - March 2018 \\ Revision - submitted May 2018 \\ Accepted - May 2018
}

\section{Abstract}

We perform Eulerian-Lagrangian simulations of solid-liquid flow in a mixing tank. The simulations are three-dimensional and time dependent and in the transitional flow regime. The lattice-Boltzmann method is used to solve the volume-averaged Navier-Stokes equations. The overall solids volume fraction is of the order of $10 \%$. Situations with the solids only partly suspended are compared to those with fully suspended solids. The emphasis is on the effect of the particle size distribution (PSD) on the suspension behavior. Four PSD's all having the same $d_{32}$ were investigated. It is harder to fully suspend particles with wider size distribution as compared to narrow distributions.

\section{Keywords}

Solid-liquid suspension, lattice-Boltzmann method, discrete particle method, particle size distribution, two-way coupling, agitated suspensions 


\section{Introduction}

Examples of agitated solid-liquid suspensions can be found in mixing tanks with the purpose of contacting solid and liquid (e.g. catalytic slurry reactors) or achieving particle formation (crystallization) or dissolution. Describing the two-phase flow in such equipment has relevance for process design and optimization since flow is determinant for inter-phase mass transfer and the rate at which surface reactions take place. Sediment transport in pipelines for various applications, such as dredging, is another prominent technological example of solid-liquid flow.

Solid-liquid flow has been the subject of many numerical simulation studies with a variety of methods and a wide spectrum of spatial and temporal resolutions. Studies of solids suspension in mixing tanks not only have relevance for processes carried out in such equipment. Since experimentation in mixing tanks is relatively easy - it is a confined system not necessarily having inlets and outlets and generally allowing for good optical access - they are a means of generating experimental data that are very useful for benchmarking numerical approaches to solid-liquid flow. Specifically experiments that make use of refractive index matching of the solids and liquid phase [1,2] are able to provide a wealth of detailed flow information that can be used for critical assessment of simulations.

In previous papers $[3,4,5]$ we have proposed a procedure for performing Eulerian-Lagrangian simulations of solid-liquid flow. It is based on solving the volume-averaged Navier-Stokes equations with a variant of the lattice-Boltzmann method, and calculating positions and velocities of individual particles through updating their equations of motion, making use of drag force correlations and soft collisions between particles. These thus are unresolved-particle simulations, as opposed to resolved-particle simulations that fully resolve the flow around individual particles and determine forces and torques on particles from first principles [6,7]. In our unresolved-particle simulations, Eulerian and Lagrangian properties communicate by means of mapping functions [5,8]. An important feature of the procedure is that it allows for the particle size to be of the order of the lattice spacing: $d=O(\Delta)$. The width of the mapping functions, i.e. the spatial extent over which Lagrangian properties are felt by the Eulerian grid 
and vice-versa, is a critical parameter in such simulations. In our procedure, making the choice for this parameter was enabled by comparing simulation results in periodic domains with particle-resolved simulations under the same conditions [4]. Applied to the solid-liquid flow in an agitated tank, the procedure was able to generate mesh-independent results [5]. That is, the method allows for a free choice of grid spacing $\Delta$ relative to particle size $d$ with $d=O(\Delta)$ so that we can keep refining the grid until it fully resolves the flow structures generated by the impeller and the way they interact with the solids.

In the current paper, the numerical procedure is extended to allow for particle size distributions. With this ability, we want to investigate how the solids suspension process in a mixing tank is affected by the size distribution and how differently sized particles are dispersed over the tank volume. We will be comparing systems with tri-disperse size distributions of various widths that all have the same volumeaverage particle size with monodisperse systems. Also different levels of suspension will be compared, including partially suspended cases where part of the particles form a (dynamic) granular bed on the bottom of the tank. With the freedom of choice for particle size relative to grid spacing our numerical procedure offers, it is well-suited for simulations involving particle size distributions.

The aim of this paper is to show the extended capabilities of our Eulerian-Lagrangian simulation procedure as well as to generate predictions on solids distributions in mixing tanks thereby inviting experimentalists to test our results. Laser sheet visualizations supported by refractive index matching would provide critical validation material for the simulation results presented here. Our flow systems have been designed -in terms of solid-over-liquid density ratio and viscosity - with a specific glass and silicone oil refractive index matched system in mind [2]. Furthermore the container has flat walls to facilitate non-deformed optical access.

The paper is organized in the following manner: We first introduce the flow system, the particle size distributions that were investigated, and the dimensionless numbers used to define specific situations. We then briefly discuss the simulation procedure (most further details are in $[3,4]$ ) with an emphasis on incorporating size distributions, and the numerical settings. We then show the start-up behavior of the 
suspension process and levels to which particles get dispersed over the tank volume as a function of flow conditions and the (width of the) size distribution. The final section summarizes the conclusions of this study.

\section{Flow system}

The flow geometry, including a coordinate system is given in Figure 1. The container is a cubic volume with side length $L$. The impeller is a 4-blade turbine with flat blades under an angle of $45^{\circ}$; it has diameter $D$. It rotates with $N$ revolutions per unit time such that it pumps liquid in the downward (i.e. negative $z$ ) direction. The liquid in the tank is Newtonian and has density $\rho$ and kinematic viscosity $v$. An impellerbased Reynolds number is defined as $\operatorname{Re}_{m x} \equiv N D^{2} / v$.

In addition to liquid, the tank contains solid, spherical particles of density $\rho_{s}>\rho$. Gravity points in the negative $z$-direction: $\mathbf{g}=-g \mathbf{e}_{\mathbf{z}}$. The total volume of solids relative to the tank volume $\left(L^{3}\right)$ is the same for every simulation in this paper. The overall solids volume fraction (solids volume over tank volume) is $\langle\phi\rangle=0.098$. The average particle diameter is $\langle d\rangle=0.0208 D$ for all simulations.

Four different particle size distributions (PSD's) have been simulated: one monosized system (all particles have diameter $d_{0}=0.0208 D$ ) that serves as the reference case, and three tri-disperse systems (i.e. systems with three different particle sizes). Their average particle diameter is defined as $\langle d\rangle=\sum_{\alpha=1}^{3} n_{\alpha} d_{\alpha}^{3} / \sum_{\alpha=1}^{3} n_{\alpha} d_{\alpha}^{2}$. The particle diameters in the three tri-disperse systems are $d=\left(d_{0} / \sqrt[3]{2}, d_{0}, \sqrt[3]{2} d_{0}\right), d=\left(d_{0} / \sqrt[3]{3}, d_{0}, \sqrt[3]{3} d_{0}\right), d=\left(d_{0} / \sqrt[3]{4}, d_{0}, \sqrt[3]{4} d_{0}\right)$ respectively. In each tri-disperse system, $50 \%$ of the solids volume is contained in the center particle size $d_{0}$, the rest in the other two particle sizes. This set of constraints to the PSD as described above (same total solids volume, same $\langle d\rangle$ etc.) gives a unique number of particles $n_{\alpha}$ per size class. The volumetric size distributions are shown in Figure 2. Actual numbers of particles and their sizes (relative to $d_{0}$ ) are in Table 1 . 
A modified Shields number $[9,10]$ has been defined as $\theta=\frac{\rho N^{2} D^{2}}{g\left(\rho_{s}-\rho\right) d_{0}}$. It is indicative for the competition between agitation and settling of solids. The two major variables in this study are the PSD (the four variants as defined above) and $\theta$. The latter has been varied in the range $16 \leq \theta \leq 260$. The dimensionless parameters that have fixed values are $\langle\phi\rangle=0.098, \operatorname{Re}_{m x}=4,000$ and $\rho_{s} / \rho=2.23$.

\section{Governing equations \& numerical procedures}

The equations solved and the numerical procedures followed are similar to [5]. The additional feature compared to [5] is that we are now dealing with particles of different sizes which has some consequences for the modeling and simulation procedures. These will be highlighted in the brief description below.

The liquid flow is governed by volume-averaged versions of the continuity equation and momentum balance $[11,12]$

$$
\begin{gathered}
\frac{\partial}{\partial t}\left(\rho \phi^{c}\right)+\nabla \cdot\left(\rho \phi^{c} \mathbf{u}\right)=0 \\
\frac{\partial}{\partial t}\left(\rho \phi^{c} \mathbf{u}\right)+\nabla \cdot\left(\rho \phi^{c} \mathbf{u u}\right)=\phi^{c} \nabla \cdot \boldsymbol{\pi}+\mathbf{f}_{\mathrm{s}}
\end{gathered}
$$

with $\phi^{c} \equiv 1-\phi$ the local continuous phase (liquid) volume fraction and $\phi$ the local solids volume fraction, $\mathbf{u}$ the interstitial liquid velocity, $\boldsymbol{\pi}$ the liquid's stress tensor, and $\mathbf{f}_{\mathrm{s}}$ the force per unit volume the solid particles exert on the liquid. Equations 1 and 2 are solved on a uniform, cubic, three-dimensional grid with a variant of the lattice-Boltzmann method [3].

Newton's equations of motion of a solid spherical particle with diameter $d_{i}$ read

$$
\begin{gathered}
\rho_{s} \frac{\pi}{6} d_{i}^{3} \frac{d \mathbf{u}_{\mathbf{p}}}{d t}=\mathbf{F}_{\mathbf{h}}+\mathbf{F}_{\mathbf{c}}-\frac{\pi}{6} d_{i}^{3}\left(\rho_{s}-\rho\right) g \mathbf{e}_{\mathbf{z}} \\
\rho_{s} \frac{\pi}{60} d_{i}^{5} \frac{d \boldsymbol{\omega}_{\mathbf{p}}}{d t}=\mathbf{T}_{\mathbf{h}}
\end{gathered}
$$

The kinematic equation is 


$$
\frac{d \mathbf{x}_{\mathbf{p}}}{d t}=\mathbf{u}_{\mathbf{p}}
$$

In Eqs. $3-5 \mathbf{u}_{\mathbf{p}}, \boldsymbol{\omega}_{\mathbf{p}}, \mathbf{x}_{\mathbf{p}}$ are the linear velocity, angular velocity and center location of the particle respectively. The particle feels a hydrodynamic force $\mathbf{F}_{h}$ and torque $\mathbf{T}_{h}$, as well as a contact force $\mathbf{F}_{c}$. Contact has two contributions: collision and lubrication.

The collisions are soft-sphere, frictionless collisions. The latter means that there is no collisional torque and that $\mathbf{F}_{\mathbf{c}}$ is a radial force. The radial repulsive soft-sphere force (i.e. a force acting on the line connecting the two sphere centers) is proportional to the overlap distance of the two spheres involved in the collision. The proportionality (i.e. spring) constant $k_{i j}$ is expressed in terms of a contact time $t_{c}$ and the masses of the particles $i$ and $j$ that collide [13]: $k_{i j}=\pi^{2}\left(m_{i}+m_{j}\right) / 2 t_{c}^{2}$ with $m_{i}=\pi \rho_{s} d_{i}^{3} / 6$.

Lubrication forces are included for representing close-range hydrodynamic interaction between particles. As for collisions, only radial forces are considered, the rationale being that they scale with $1 / s$ whereas tangential effects (including torque) show the weaker scaling with $\ln s$ with $s$ the space between two particle surfaces [14]. The expression we use for the radial lubrication force component between two particles $i$ and $j$ is [15]

$$
F_{l u b}=\frac{3}{4} \pi \rho v \frac{d_{i}^{2} d_{j}^{2}}{\left(d_{i}+d_{j}\right)^{2}}\left(\frac{1}{s}-\frac{1}{s_{0}}\right)\left(\mathbf{n} \cdot \Delta \mathbf{u}_{\mathbf{i j}}\right) \text { if } s \leq s_{0}, \text { and } F_{l u b}=0 \text { if } s>s_{0}
$$

with $\mathbf{n} \cdot \Delta \mathbf{u}_{\mathrm{ij}}$ the relative velocity between the two particles along the line connecting their centers, and $s_{0}$ a model parameter that denotes the distance below which the lubrication force becomes active. A second model parameter $s_{1}$ is the (smaller) distance below which the lubrication force saturates [16]. It prevents the lubrication force from diverging when $s$ approaches zero and accounts for surface roughness of the particles. The parameters were set to $s_{0}=0.1\left(d_{i}+d_{j}\right) / 2$ and $s_{1}=0.001\left(d_{i}+d_{j}\right) / 2$. The lubrication force is attractive upon separation, and repulsive upon approach of particles. 
As previously [5], for the hydrodynamic force we have taken a drag-only approach, i.e. lift, added mass, stress-gradient, and history forces [17] have been discarded. The drag force is written as

$$
\mathbf{F}_{\mathbf{D}}=3 \pi \rho v d_{i}\left(\mathbf{u}-\mathbf{u}_{\mathbf{p}}\right) F(\operatorname{Re}, \phi)
$$

with $\operatorname{Re}=(1-\phi)\left|\mathbf{u}-\mathbf{u}_{\mathbf{p}}\right| d_{i} / \nu$. The function $F$ is partitioned as $F(\operatorname{Re}, \phi)=p(\operatorname{Re}) q(\phi)$ with $p(\operatorname{Re})=\left(1+0.15 \operatorname{Re}^{0.687}\right)$ the Schiller-Naumann correlation [18], and $q(\phi)=(1-\phi)^{-2.65}$ the Wen \& Yu expression [19]. The local solids volume fraction $\phi$ in the above expressions is the result of particles of all sizes. We thus apply - in an ad hoc manner [20] - notions of how drag depends on particle size and local porosity for monodisperse systems to polydisperse systems by replacing $d$ with $d_{i}$. The Wen $\& \mathrm{Yu}$ correlation has been applied as it is well suited for solid-liquid systems with relatively small particle Stokes numbers [21].

The hydrodynamic force $\mathbf{F}_{\mathbf{h}}$ as it is contained in Eq. 3 is related to the drag force according to $\mathbf{F}_{\mathbf{h}}=\mathbf{F}_{\mathbf{D}} /(1-\phi)$ [22]. The drag force $\mathbf{F}_{\mathbf{D}}$ is mapped on the Eulerian mesh to determine the body force $\mathbf{f}_{\mathbf{s}}$ in Eq. 2. For mapping, the clipped fourth-order polynomial function has been used [23]. Benchmark simulations [4] of monodisperse systems with particle diameter $d$ have led to the width of the mapping function $\lambda=1.5 d$ as an optimal choice. This choice we apply to the polydisperse cases as well, i.e. the width of the mapping function associated to a particle with diameter $i$ is $\lambda_{i}=1.5 d_{i}$.

Particles rotate under the influence of the hydrodynamic torque $\mathbf{T}_{\mathbf{h}}=\pi \rho v d_{i}^{3}\left(\frac{1}{2} \boldsymbol{\omega}-\boldsymbol{\omega}_{\mathbf{p}}\right)$ with $\boldsymbol{\omega}$ the vorticity of the liquid in the direct vicinity of the particle. Particle rotation is not coupled back to the liquid and therefore has no influence on the overall dynamics of the two-phase flow in the mixing tank.

The default resolution of the simulations is such that the average particle diameter is $\langle d\rangle=d_{0}=1.6 \Delta$. Then the impeller has diameter $D=76.8 \Delta$ and the tank side length $L=176 \Delta$. The impeller makes one revolution in 2560 time steps. The kinematic viscosity is chosen as to match the desired impeller-based Reynolds number $\left(\operatorname{Re}_{m x}=4,000\right)$. With an impeller-based Reynolds number of 
4,000 and the current levels of spatial and temporal resolution there is no need for turbulence modeling through subgrid-scale models as used in large-eddy simulations [5]. Gravitational acceleration is set to achieve a certain value of $\theta$. The collision time $t_{c}$ is set to 10 time steps which corresponds with the time needed for $1.4^{\circ}$ of impeller rotation. When presenting the results we will briefly discuss the subject of grid convergence: we compare tri-disperse results with the default resolution of $\langle d\rangle=d_{0}=1.6 \Delta$ with those having $\langle d\rangle=d_{0}=2.0 \Delta$. The subject of grid convergence was extensively discussed - for monosized systems - in [5].

\section{Results}

\section{Impressions \& start-up behavior}

Initially the particles form a loosely packed granular bed resting on the tank bottom. The solids volume fraction of this bed is approximately 0.60 . The beds are generated by randomly placing the particles - in a non-overlapping fashion - in a container of size $L \cdot L \cdot 0.45 L(x \cdot y \cdot z)$. Random numbers are taken from a uniform distribution representing the $x, y$ and $z$ location of particle centers. The particle location is accepted if the new particle does not overlap with already placed particles; it is rejected if the new particle overlaps. This process is repeated until the desired number of particles per particle size has been placed. This process has limits to the solids fractions that can be reached [24]. As an example, for particle size distribution (PSD) \#4 (see Table 1), a solids volume fraction of approximately 0.46 can be reached this way. A cross section through this system is shown in Figure 3a. Letting the particles in this system settle under gravity through the same liquid as in the mixing tank creates the loosely packed bed as shown in Figure 3b. When a less dense randomly-placed system is generated, as in Figure 3c, the eventual settled granular bed has a different structure; there is a profound level of segregation with many small particles at the bed's upper surface. We will be comparing mixing tank simulations with a mixed granular bed (Figure 3b) and a segregated granular bed (Figure 3d) as a starting point and assess for how long the agitated systems remembers the initial structure of the bed. 
The so generated beds are placed in the mixing tank. Liquid and particles initially have zero velocity. At time equals zero $(t=0)$ we start the impeller with its angular velocity $\Omega$ gradually increasing according to $\Omega=\pi N\left[1-\cos \left(\frac{1}{2} t N \pi\right)\right]$ in the period $0 \leq t \leq 2 / N$; for $t>2 / N$ the angular velocity of the impeller is constant at $\Omega=2 \pi N$. In the start-up period with length $2 / N$ the impeller completes one revolution, after that it runs with constant speed. With the bed as shown in Figure $3 \mathrm{~d}$ as initial condition, the start-up of the agitation process is visualized in Figure 4. One sees the formation of a downward liquid stream generated by the impeller impacting on the granular bed. That bed is eroded and particles start to get suspended. A more detailed view of the erosion process is shown in Figure 5 where in a vertical cross section through the center of the tank - information on particle locations and liquid velocity vectors are combined. The right panel of Figure 5 shows that small particles collect underneath the impeller for the case of the initially segregated bed.

The way the suspension process evolves in time is summarized in Figure 6, that also gives an overview of the main simulation cases that have been studied in this paper. The time series show the evolution of the average vertical location of the particles in the period $0 \leq t \leq 60 / N$. Three levels of agitation are shown in the figure (Shields numbers $\theta=260,65.0$, and 16.2) as well as five different initial granular beds: one bed with PSD \#2; one mixed bed with PSD \#3; one segregated bed with PSD \#3; one mixed bed with PSD \#4; one segregated bed with PSD \#4. In the time series we distinguish between the overall, volume-average vertical location of the solids, and the average vertical location of the individual particle sizes (three per PSD). In addition we show - for reference - data for monosized systems.

For the highest Shields number of $\theta=260$, the particles distribute themselves over the entire height of the tank, such that in steady state their average vertical location $\left\langle z_{p}\right\rangle \approx L / 2$. Compared to the monosized system, the development to steady state for the polydisperse systems takes longer (approximately 40 revolutions for monosized and 50 revolutions for polydisperse). It is also interesting to 
note that, consistently, the largest particles have the highest $\left\langle z_{p}\right\rangle$, also when they start on average lowest in the segregated initial granular bed cases.

As expected, lowering the Shields number clearly reduces $\left\langle z_{p}\right\rangle$. We now see the largest particles on average - ending up at lower levels in the tank, and the smallest particles highest. There is a dependency on the initial bed structure that persists over all 59 impeller revolutions simulated which is due to the fact that for the lower Shields numbers not all particles get mobilized. This specifically is the case for $\theta=16.2$.

For all polydisperse cases studied, the volume-averaged vertical location of the solids closely follows the average location of the mid-size particles, where it should be reminded that the mid-size particles make up half of the total solids volume (see Figure 2 and Table 1). After steady state is reached, there is no significant / consistent difference between the volume-averaged vertical location of the polydisperse systems and that of the monosized systems.

In the previous paper that dealt with monosized systems exclusively [5], grid convergence of the numerical procedure was demonstrated. In the present paper grid effects have been investigated by comparing simulations with $d_{0}=1.6 \Delta$ to those with $d_{0}=2.0 \Delta$ having for the rest the same dimensionless parameters. The results in Figure 7 indicate no significant differences in terms of $\left\langle z_{p}\right\rangle$ for the two resolutions. Similar differences were observed when comparing monosized systems with $d=1.6 \Delta$ and $d=2.0 \Delta$ in [5].

\section{Developed flow and steady-state behavior}

In this sub-section the focus is on the stages of the solids suspension process when the flow has fully developed. In Figure 8 we show instantaneous realizations of how the particles disperse themselves over the tank volume for PSD \#4 as well as liquid velocity magnitude distributions for three values of the modified Shields number. For the lowest value $(\theta=16.2)$ the particles get hardly entrained by the liquid 
flow. The main effect of the liquid flow is to deform the bed shape and to erode its surface so that only a small number of the smaller particles get detached from the bed. This has been observed for the other PSD's at $\theta=16.2$ as well and in the remainder of this section we will not discuss results with this Shields number any further. The velocity contours show the liquid stream coming off the impeller and it being deflected in case dense packings of solids form underneath the impeller.

Since the suspension process is competition between gravity and fluid flow, the way particles on average distribute themselves vertically is considered. With PSD \#4 as an example, this is shown in Figure 9. The solids concentration profiles have been scaled such that a perfectly uniform distribution would show $\phi /\langle\phi\rangle=1$ for all $z$. For $\theta=260$ there is not much difference between the way the differently sized particles distribute over the vertical $(z)$ coordinate, despite the fact that a large particle has a volume that is 16 times that of a small particle. On average, the larger particles are slightly over-represented in the upper parts of the tank. The latter is a result of the larger particles being more inertial and as a result able to store more kinetic energy, see Figure 10. The Stokes number - as a measure for particle inertia - of the three particle sizes in PSD \#4 are St $\equiv \frac{2}{9} \frac{\rho_{s}}{\rho} \frac{d_{i}^{2} 4 N}{v}=1.4,3.4$ and 8.9 respectively (in the definition of the St we take the blade passage time $(4 N)^{-1}$ as the flow time scale). The higher kinetic energy of the larger particles make them disperse better and therefore a little less susceptible to settling in the upper regions of the tank where the liquid flow is relatively weak.

The vertical concentration profiles for $\theta=260$ do not depend on the initial state of the granular bed (mixed versus segregated). For $\theta=260$ the results indicate that we have a well-mixed flow system with well-suspended solids. For $\theta=65$ the situation is clearly different with now relatively less larger particles in the upper part of the tank and an overrepresentation of them near the bottom. Also the initial bed configuration still has an impact on the vertical concentration profile 50 to 60 impeller revolutions after startup with higher concentrations of large particles close to the bottom wall for the initially segregated bed. 
An interesting effect of the size distribution of the solids on the overall behavior of the suspension process is the formation and size of a persistent solids cone underneath the impeller. The liquid stream coming off the impeller in the downward direction is not very well able to agitate the region near the center of the tank underneath the impeller. As a result, the granular bed there is hard to mobilize and particles collect in this relative quiescent part of the tank. In Figure 11 we visualize this effect by showing for a number of simulations the time-averaged solids volume fraction (of all particle types combined) in a vertical cross section through the center of the tank. The monosized system at $\theta=260$ hardly shows a solids cone underneath the impeller and that also holds for PSD \#2. For the wider size distributions PSD \#3 and \#4, however, a small solids cone does form on the bottom underneath the impeller. Differently from expected, the latter cone does not only consist of the large particles, it also contains the smaller ones as can be seen in Figure 12. For the lower value of $\theta=65$, a solids cone is present for all size distributions, see the right panels of Figure 11. The cone is larger for the wider PSD's. As for $\theta=260$, all types of particles are present in the cone, see Figure 12. The cone is a manifestation of the mutual feedback between the solid and liquid dynamics. Velocity fields of liquid and solid in the cone region are shown in Figure 13. For $\theta=65$ it is remarkable to see the packing of solids in the cone being exposed to a strong liquid shear flow at its sloped surface.

\section{Conclusions}

In this paper we have described and applied a numerical modeling procedure for polydisperse solid-liquid flow based on a Eulerian-Lagrangian approach. Polydispersity enters the procedure at a number of places: in the short-range interactions between particles (lubrication and collisions), in the way the drag force on the particles is determined, as well as in the transfer between Eulerian (liquid) and Lagrangian (particles) information through mapping function, the width of which depends on particle size. Since the numerical procedure for monosized systems has previously shown to give results that are independent of particle 
size relative to grid spacing, it is argued that the extended procedure is well placed for accommodating situations with multiple particle sizes in a single simulation.

In order to focus on particle size distribution effects, we compare cases that have the same volumeaverage particle size and only differ in terms of the width of the size distribution. With the simulations, we go through the entire process of solids suspension, i.e. we begin with a loosely packed, static granular bed on the bottom of a mixing tank and then start impeller rotation that induces fluid flow and subsequently mobilization of the solids. The structure of the granular bed the simulation starts with, i.e. the way the different particle sizes are distributed over the bed height, has an influence long after the impeller was switched on, specifically when the flow is not sufficiently strong to achieve full suspension of solids. It was also noted that, compared to monosized systems, it takes more time to reach a steady suspension state for polydisperse systems.

In terms of global characteristics - for instance the average height of the particles in the tank - the volume-average trends of polydisperse systems closely follow their monodisperse counterparts. At a more detailed level, however, important differences in suspension behavior between different size distributions are observed, such as the formation and extent of a cone of solids resting on the bottom near the center of the tank that depends on the width of the size distribution.

It is very important to critically assess the predictions as presented in this paper that are based on numerical simulations only. Not only because strong assumptions have been made regarding the physics of the system (for instance drag-only when it comes to hydrodynamic forces), but also because of limits on the spatial resolution with which the liquid flow is simulated. Experimental work that reveals details about the way solids get distributed over the container, as well as solids and liquid flow velocities is much needed for validation purposes. Optical experiments based on refractive index matching would be ideally suited for this purpose. We are developing an experiment in a lab-scale square tank with a side-length of $220 \mathrm{~mm}$. With the geometrical settings of this paper, the particles would then have an average size of $\langle d\rangle=d_{0} \approx 2 \mathrm{~mm}$. The refractive index matched system in [2] uses glass particles and a specific silicon oil 
with a dynamic viscosity of $0.021 \mathrm{~Pa} \cdot \mathrm{s}$. An impeller speed of $N=9.2 \mathrm{rev} / \mathrm{s}$ would achieve flow conditions (as quantified by Reynolds and Shields numbers) in line with the simulations presented in the current paper. 


\section{References}

[1] M.M. Cu, R.J. Adrian, Refractive index matching and marking methods for highly concentrated solid-liquid flows, Exp. Fluids 22 (1997) 261-264.

[2] G. Li, Z. Gao, Z. Li, J. Wang, J.J. Derksen, Particle-resolved PIV experiments of solid-liquid mixing in a turbulent stirred tank, AIChE J. 64 (2018) 389-402.

[3] R. Sungkorn, J.J. Derksen, Simulations of dilute sedimenting suspensions at finite-particle Reynolds numbers, Phys. Fluids 24 (2012) 123303.

[4] J.J. Derksen, Assessing Eulerian-Lagrangian simulations of dense solid-liquid suspensions settling under gravity, Comp. \& Fluids in press DOI 10.1016/j.compfluid.2016.12.017 (2016).

[5] J.J. Derksen, Eulerian-Lagrangian simulations of settling and agitated dense solid-liquid suspensions - achieving grid convergence, AIChE J. 64 (2018) 1147-1158.

[6] M. Uhlmann, Interface-resolved direct numerical simulation of vertical particulate channel flow in the turbulent regime, Phys. Fluids 20 (2008) 053305.

[7] J.J. Derksen, Direct numerical simulations of aggregation of monosized spherical particles in homogeneous isotropic turbulence, AIChE J. 58 (2012) 2589-2600.

[8] J. Capecelatro, O. Desjardins, An Euler-Lagrange strategy for simulating particle-laden flow, J. Comp. Phys. 238 (2013) 1-31.

[9] A. Shields, Anwendung der Aenlichkeitsmechanik und der Turbulenzforschung auf die Geschiebebewegung, Mitteilungen der Preussischen Versuchsanstallt fuer Wasserbau und Schiffbau, Berlin, Heft 26 (1936) downloaded from http://repository.tudelft.nl/view/hydro/uuid\%3A61a19716-a994-4942-9906-f680eb9952d6/ on April $21,2015)$.

[10] J.J. Derksen, Highly resolved simulations of solids suspension in a small mixing tank, AIChE J. 58 (2012) 3266-3278.

[11] K. Sankaranarayanan, S. Sundaresan, Lattice Boltzmann simulation of two-fluid model equations, Ind. Eng. Chem. Res. 47 (2008) 9165-9173.

[12] D. Gidaspow, Multiphase Flow and Fluidization, Academic Press, San Diego (1994).

[13] G.J. Rubinstein, J.J. Derksen, S. Sundaresan, Lattice-Boltzmann simulations of low-Reynolds number flow past fluidized spheres: effect of Stokes number on drag force, J. Fluid Mech. 788 (2016) 576-601.

[14] S. Kim, S.J. Karrila, Microhydrodynamics: Principles and selected applications, ButterworthHeinemann, Boston (1991). 
[15] N-Q. Nguyen, A.J.C. Ladd, Lubrication corrections for lattice-Boltzmann simulations of particle suspensions, Phys. Rev. E 66 (2002) 046708.

[16] J.J. Derksen, S. Sundaresan, Direct numerical simulations of dense suspensions: wave instabilities in liquid-fluidized beds, J. Fluid Mech. 587 (2007) 303-336.

[17] M.R. Maxey, J.J. Riley, Equation of motion for a small rigid sphere in a nonuniform flow, Phys. Fluids 26 (1983) 883-889.

[18] L. Schiller, A. Naumann, Uber die grundlagenden Berechnungen bei der Schwerkraftaufbereitung, Ver. Deut. Ing. Z. 77 (1933) 318-320.

[19] C.Y. Wen, Y.H. Yu, Mechanics of fluidization, Chem. Engng. Prog. 62 (1966) 100-111.

[20] M.A. van der Hoef, R. Beetstra, J.A.M. Kuipers, Lattice-Boltzmann simulations of low-Reynoldsnumber flow past mono- and bidisperse arrays of spheres: results for the permeability and drag force, J. Fluid Mech. 528 (2005) 233-254.

[21] G.J. Rubinstein, J.J. Derksen, S. Sundaresan, LatticeBoltzmann simulations of low-Reynolds number flow past fluidized spheres: effect of Stokes number on drag force, J. Fluid Mech. 788 (2016) 576601.

[22] R. Beetstra, M.A. Van der Hoef, J.A.M. Kuipers, Drag force of intermediate Reynolds number flow past mono- and bi-disperse arrays of spheres, AIChE J. 53 (2007) 489-501.

[23]N.G. Deen, M. Van Sint Annaland, J.A.M Kuipers, Multi-scale modeling of dispersed gas-liquid two-phase flow, Chem. Eng. Sc. 59 (2004) 1853-1861.

[24]S. Torquato, T.M. Truskett, P.G. Debenedetti, Is random close packing of spheres well defined?, Phys. Rev. Lett. 84 (2000) 2064-2067. 
Figures

Figure 1. Mixing tank geometry: top view and side view. The origin of the Cartesian coordinate system is in the center of the bottom wall.
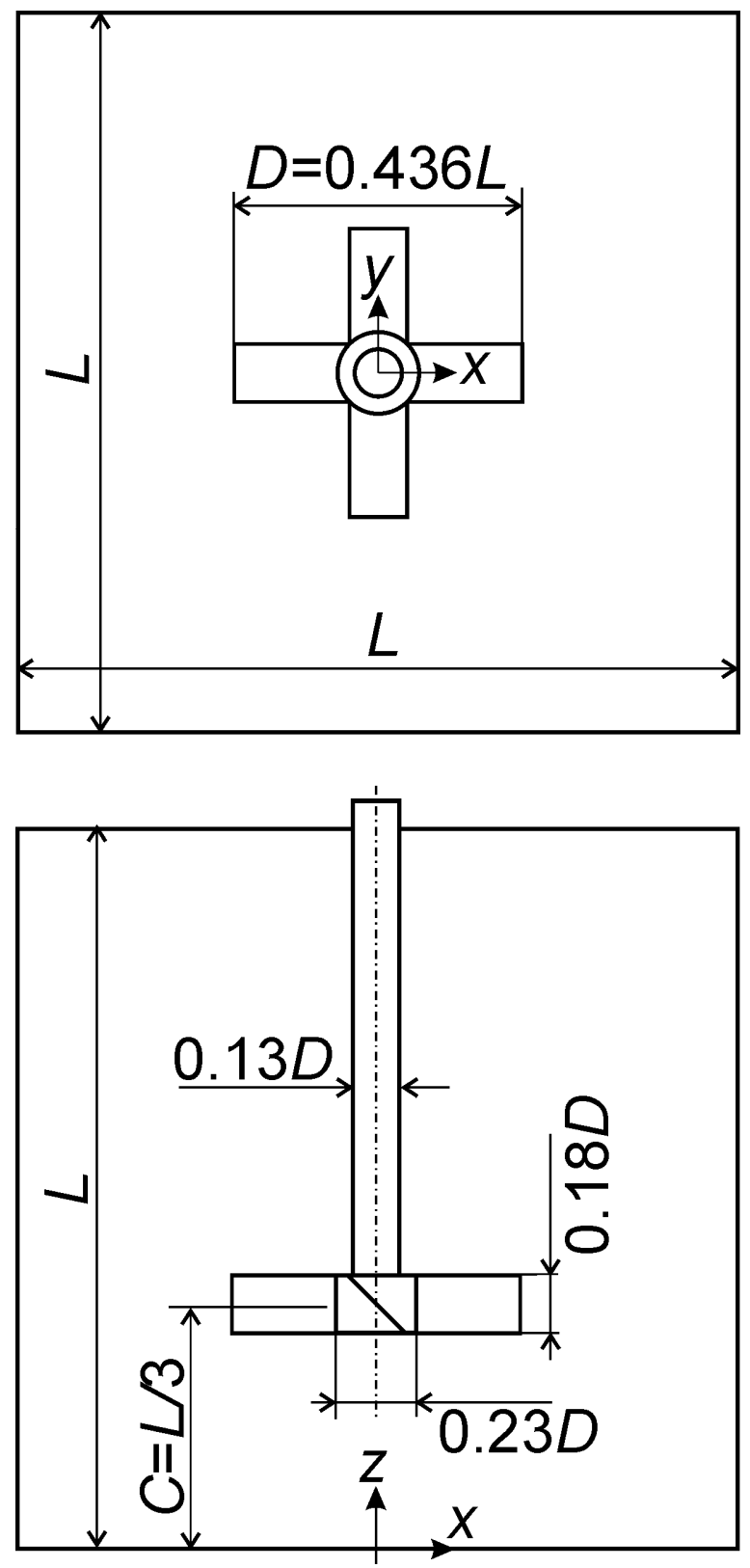
Figure 2. Schematic of the four particle size distributions. Black: monosized; blue: particle sizes $\left(d_{0} / \sqrt[3]{2}, d_{0}, \sqrt[3]{2} d_{0}\right)$; red: $\left(d_{0} / \sqrt[3]{3}, d_{0}, \sqrt[3]{3} d_{0}\right)$; green: $\left(d_{0} / \sqrt[3]{4}, d_{0}, \sqrt[3]{4} d_{0}\right)$. All distributions have $\langle d\rangle=d_{0}$; all have the same total volume of solids, the three tri-disperse distributions have $50 \%$ of their volume in particles with diameter $d_{0}$.

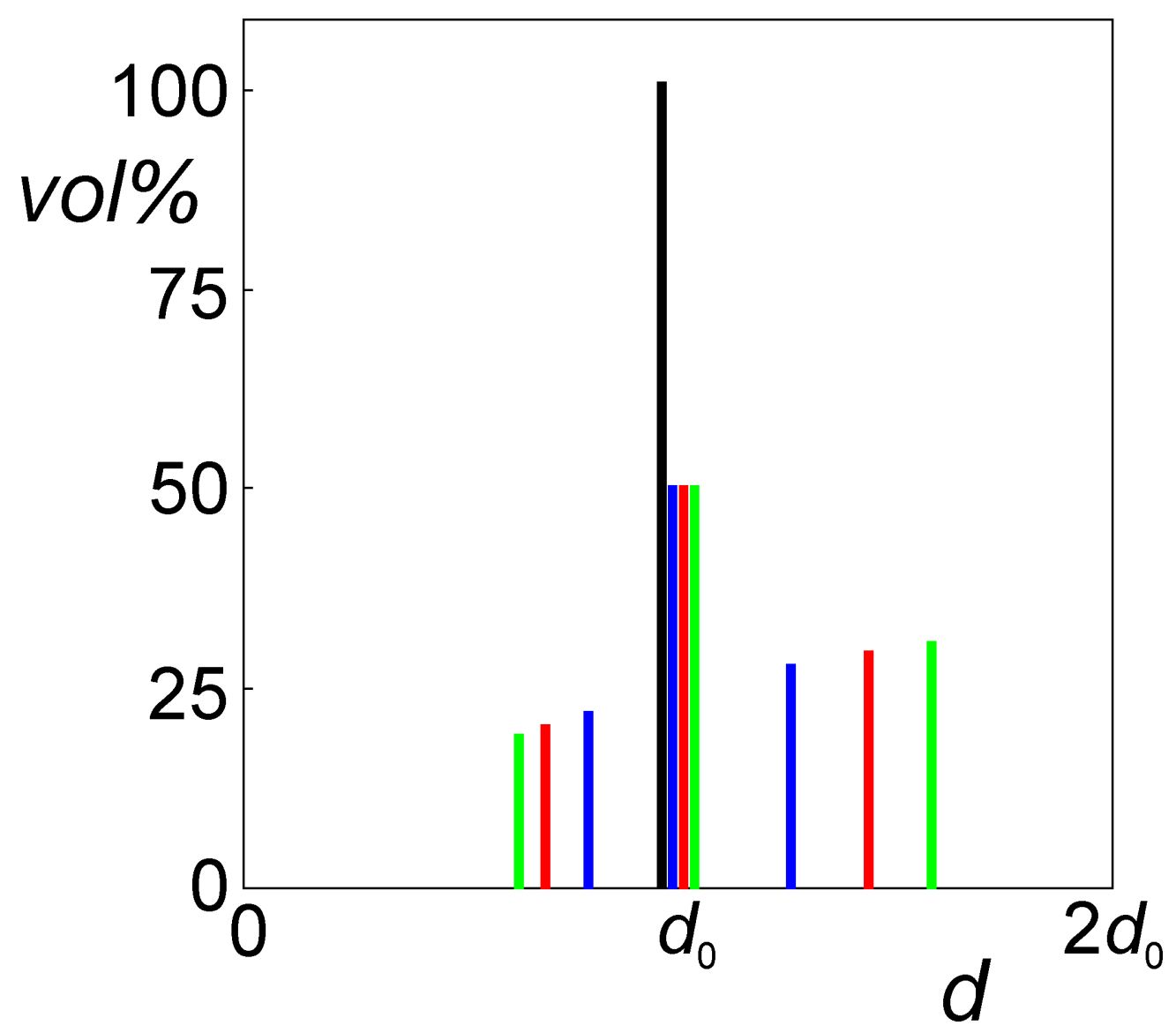


Figure 3. Creating granular beds with PSD \#4. a. Cross section through a set of non-overlapping particles with random positions; b. the same set of particles after settling; c. a less dense set of particles with random positions; d. the resulting granular bed after settling. Large, middle and small particles are red, green, and blue respectively.

\section{a}

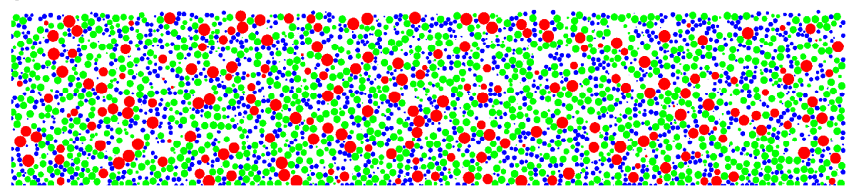

$b$

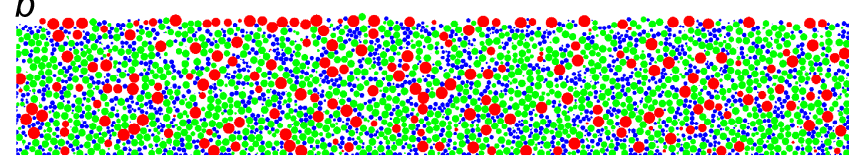

$c$

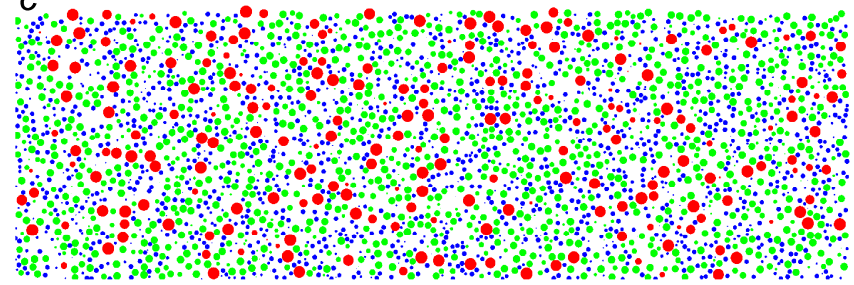

ஸै $d$

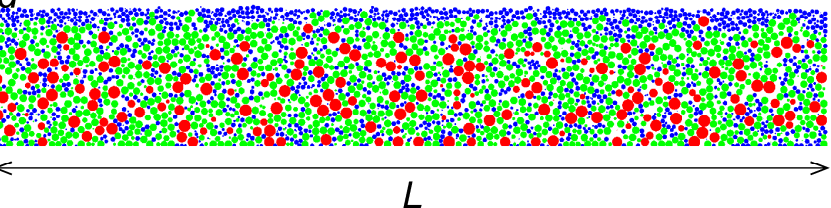


Figure 4. Three snapshots of the suspension process with PSD \#4 $\left(d_{0} / \sqrt[3]{4}, d_{0}, \sqrt[3]{4} d_{0}\right)$. The contours in the vertical plane through the center of the tank denote the interstitial liquid velocity magnitude. From small to large the particle colors are blue, yellow, and red. Only the particles in and behind the contour plane are depicted. $d_{0}=1.6 \Delta, \theta=260$. Since the first impeller revolution takes $2 / N$, the three snapshots are after 2.0, 4.0 and 8.1 impeller revolutions respectively.
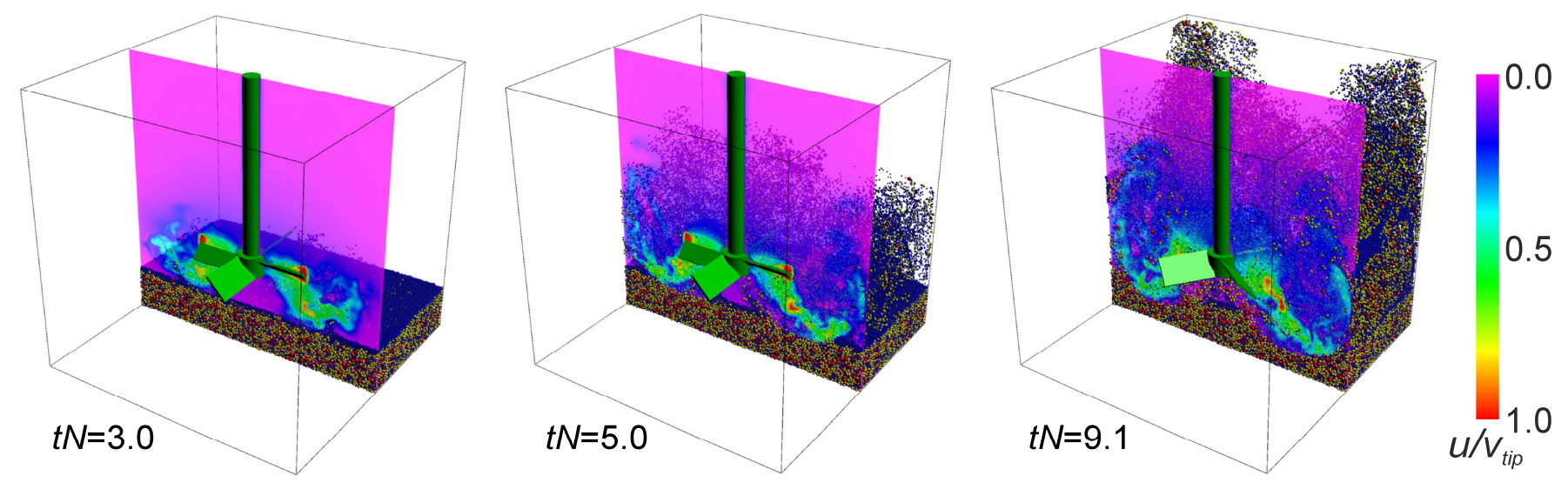
Figure 5. Cross sections through the granular beds (particle locations and liquid interstitial velocity vectors) of two simulations with PSD \#4. Left a mixed bed; right a segregated bed. Moment $t N=9.1$, $d_{0}=1.6 \Delta, \theta=260$.

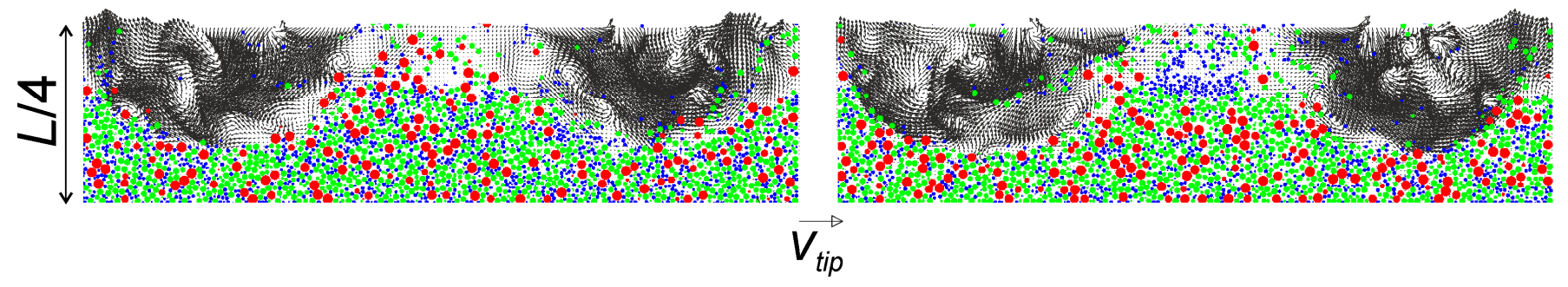


Figure 6. Time series of the average vertical particle positions $\left\langle z_{p}\right\rangle$ as a function of time. From left to right $\theta$ decreases. Three different PSD's; $m x$ and seg stands for mixed and segregated bed as initial condition; mono is the time series for monosized particles at the indicated $\theta$; vol av is volume averaged; small, mid, large are the three particles sizes per PSD. $d_{0}=1.6 \Delta$.
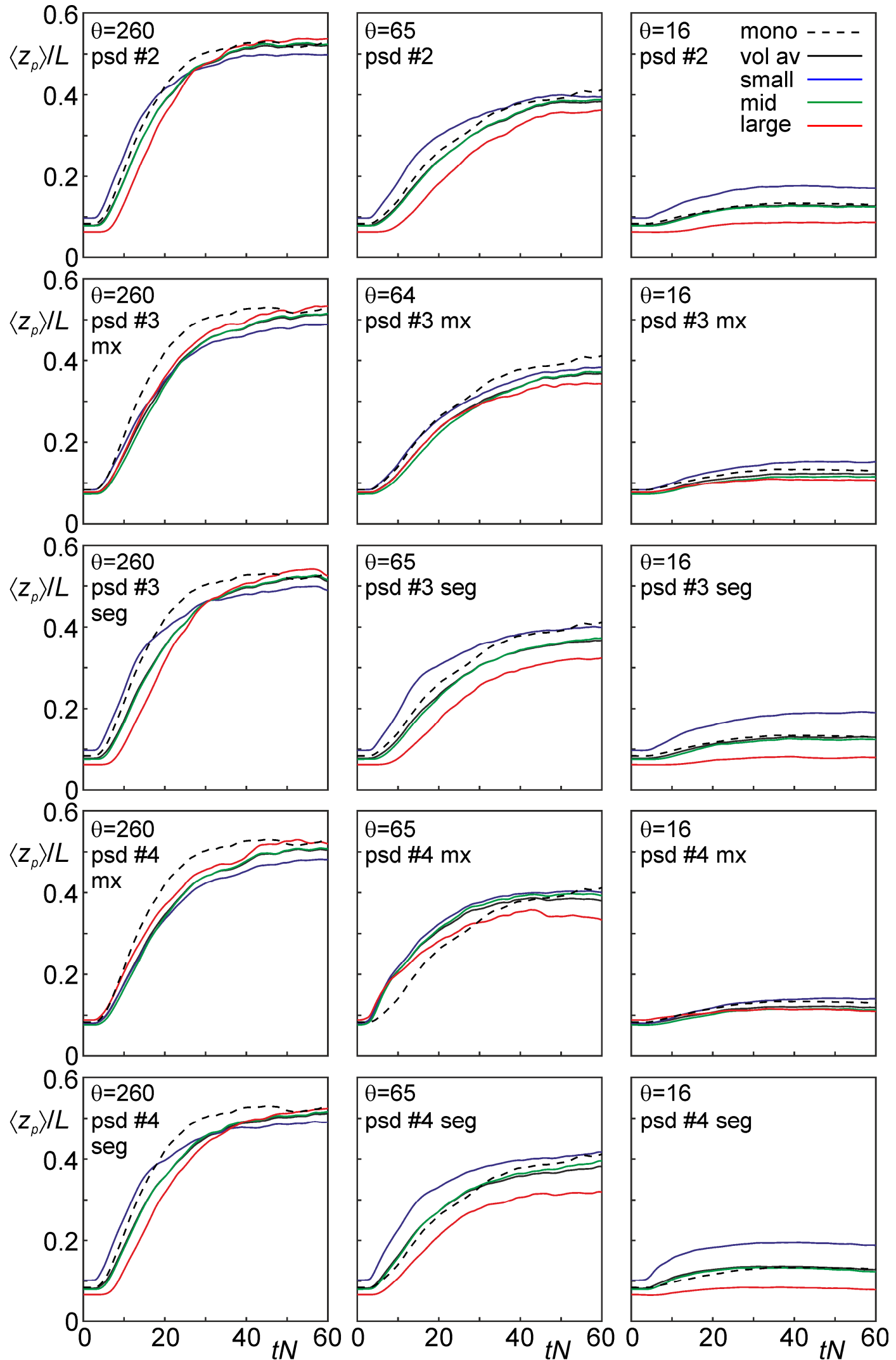
Figure 7. Time series of the average vertical particle positions $\left\langle z_{p}\right\rangle$ as a function of time. Assessment of grid effects. The solid curves have the default resolution of $d_{0}=1.6 \Delta$, the dashed curves have $d_{0}=2.0 \Delta$. Left: PSD \#3 with initially segregated bed at $\theta=260$; right: PSD \#3 with segregated bed at $\theta=16.2$. Blue, green and red refer to the small, middle and large particles in the PSD respectively.

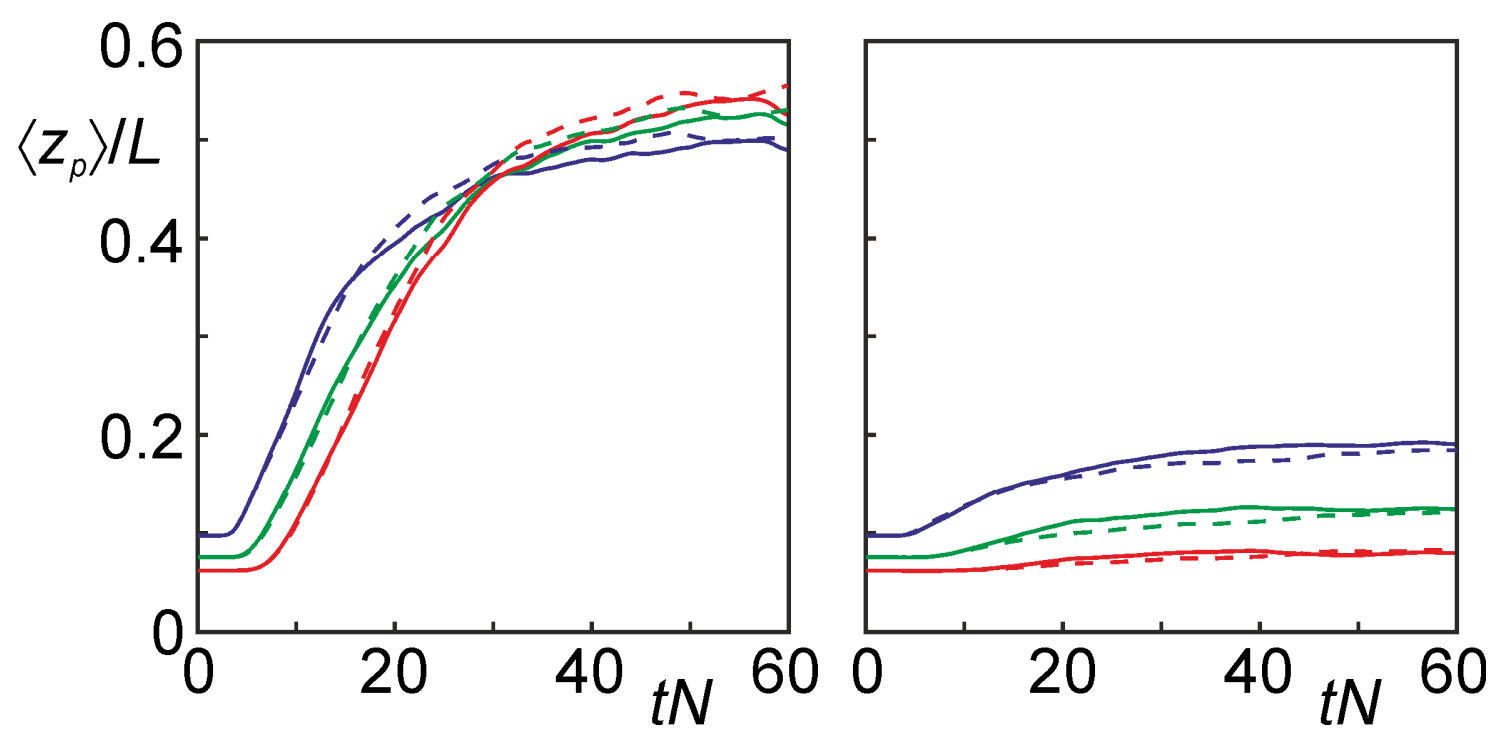


Figure 8. Instantaneous realizations at $t N=60$ with PSD \#4. Contours of interstitial liquid velocity magnitude and particles in a vertical cross section through the center of the mixing tank. From left to right: $\theta=260,65.0,16.2$. Large, middle, and small particles are black, grey, and white respectively. $d_{0}=1.6 \Delta$.
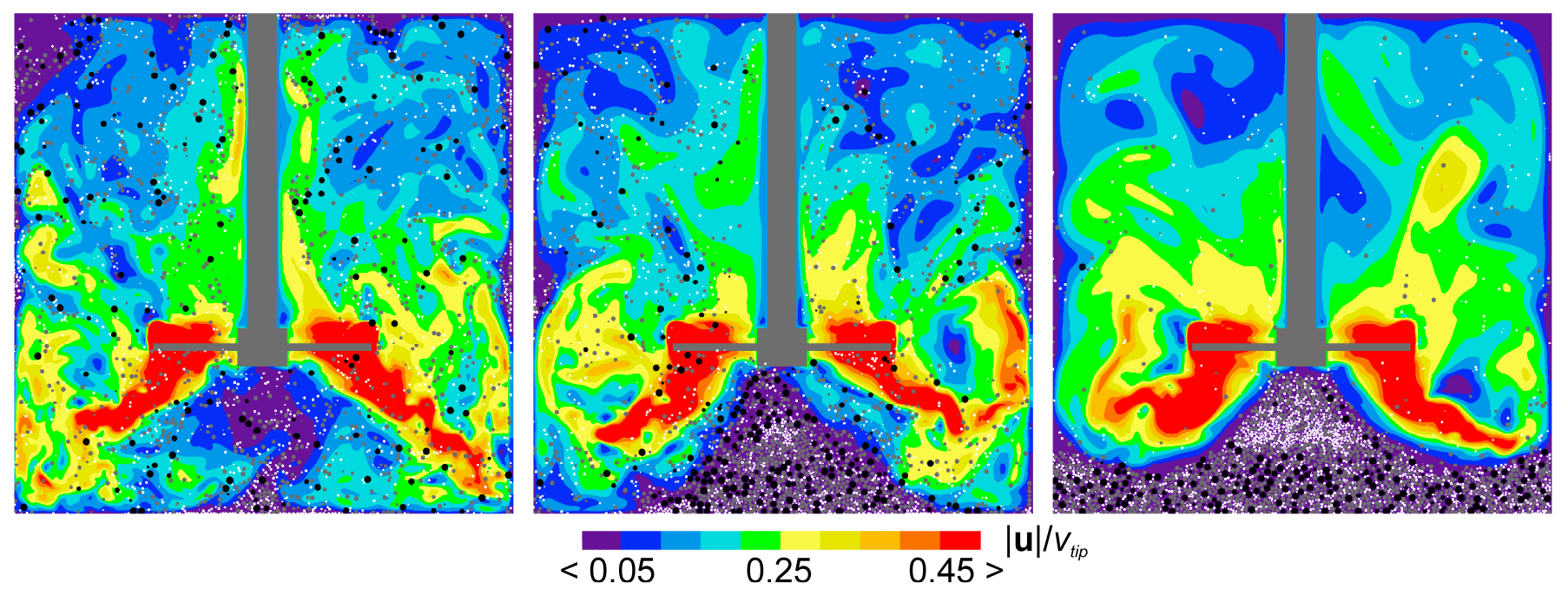
Figure 9. Time-averaged (over $50 \leq t N \leq 60$ ) vertical particle concentration profiles for all particles and for individual particle sizes normalized by the (per-particle-size) tank-averaged concentration $\langle\phi\rangle$ for PSD \#4 with mixed bed initial condition (top), and segregated bed initial condition (bottom). $\theta$ as indicated; $d_{0}=1.6 \Delta$.
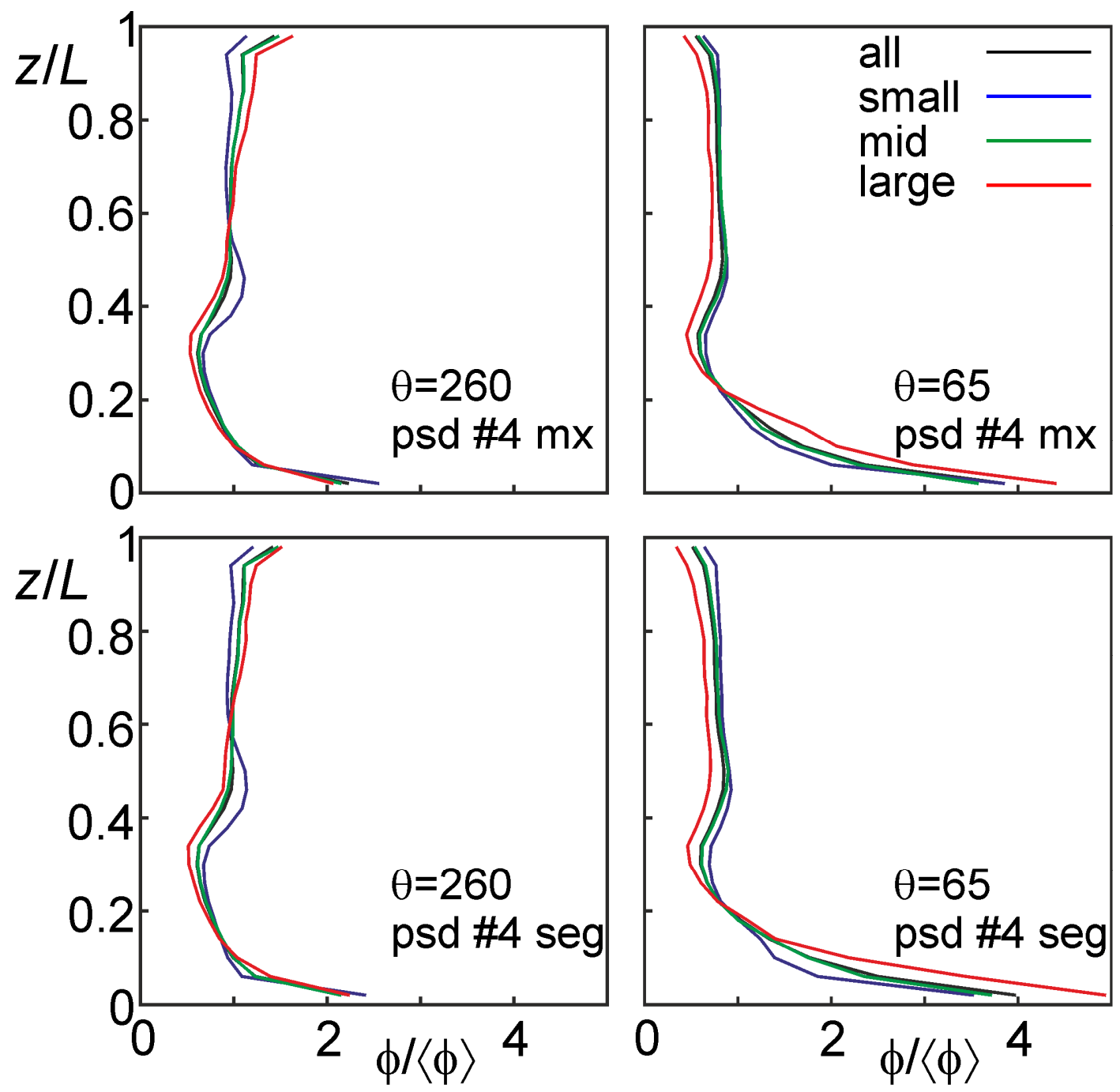
Figure 10. Time-averaged (over $50 \leq t N \leq 60$ ) vertical profiles of kinetic energy of particles $k$ normalized by $k_{0} \equiv \frac{1}{2} m_{0} \mathrm{v}_{\text {tip }}^{2}$ with $m_{0}$ the mass of a particle with diameter $d_{0} ; d_{0}=1.6 \Delta$.

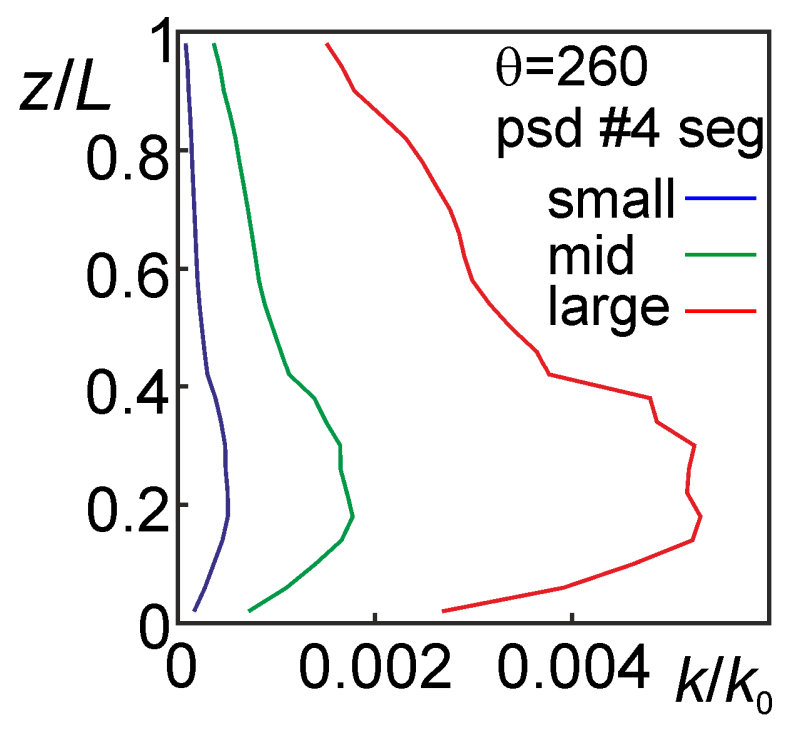


Figure 11. Time-averaged (over $50 \leq t N \leq 60$ ) solids volume fraction contours in a vertical plane through the center of the mixing tank. Left: $\theta=260$; right: $\theta=65.0$. From top to bottom: monosized system (PSD \#1), PSD \#2, PSD \#3 with mixed initial bed, PSD \#4 with mixed initial bed. $d_{0}=1.6 \Delta$. The white rectangles in the lower panels indicate the fields of view in Figure 12.
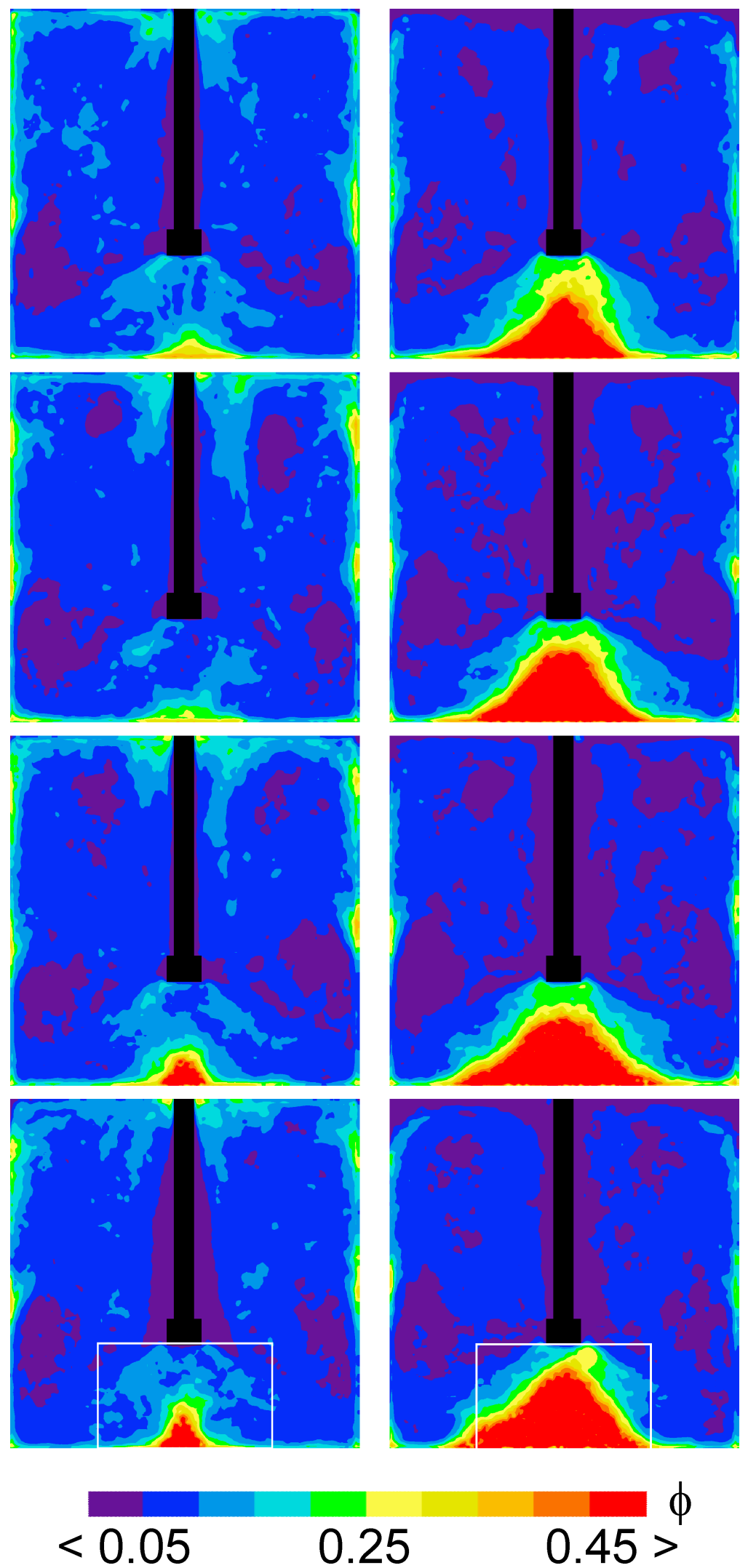
Figure 12. Instantaneous particle location realizations at $t N=60$ for PSD \#4 in the plane and field of view indicated in Figure 11 (lower panels). Left: $\theta=260$; right $\theta=65.0 . d_{0}=1.6 \Delta$.
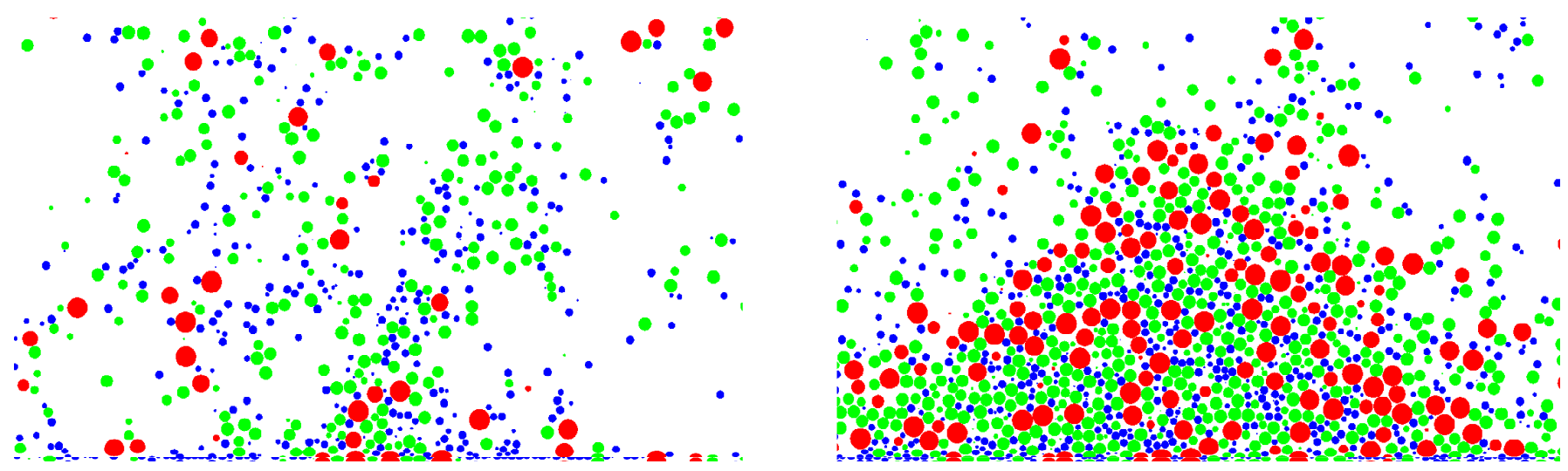
Figure 13. Instantaneous particle velocity vectors (top) and interstitial liquid velocity vectors (bottom) at $t N=60$ for PSD \#4 around the solids cone that forms on the bottom. Left: $\theta=260$; right $\theta=65.0$. For the top panels: the color of the vector indicates the particle size (blue, green, and red for small, middle, and large particles respectively). $d_{0}=1.6 \Delta$.

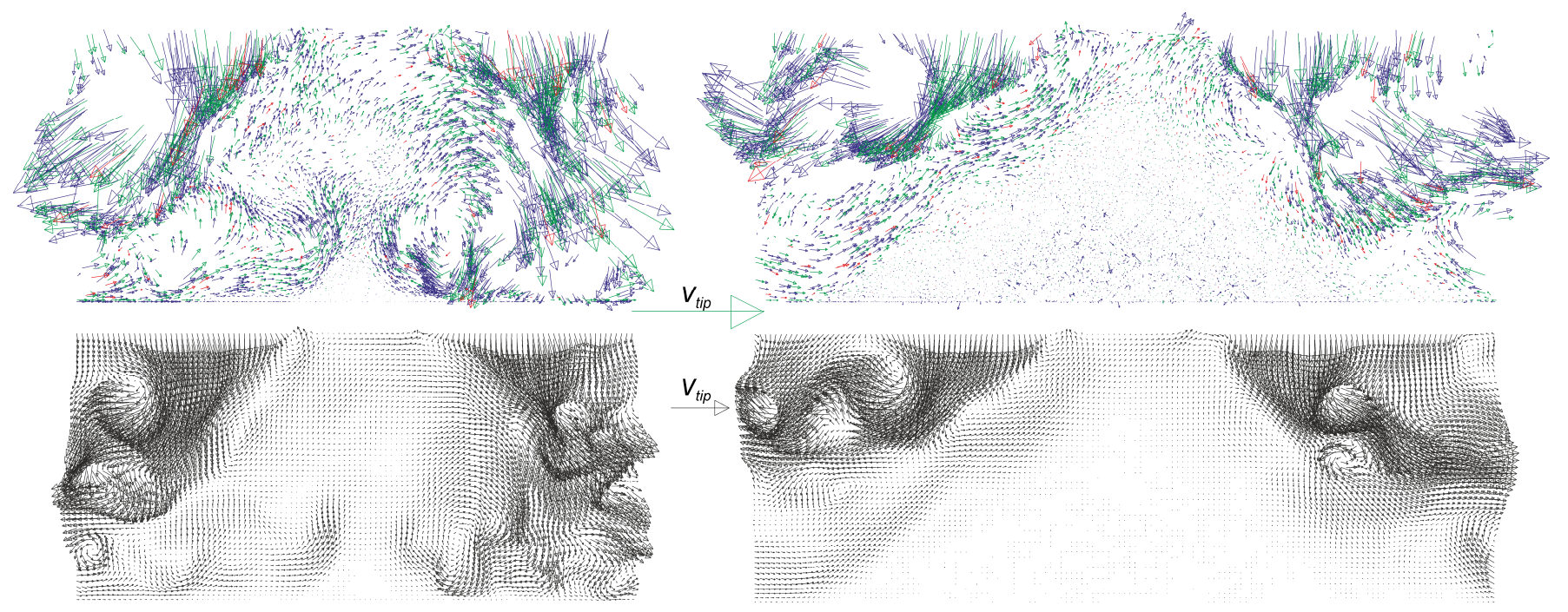


Tables

Table 1. Particle size distributions

\begin{tabular}{|l|l|l|l|}
\hline Size distr. \# & & & \\
\hline 1 & $d=d_{0}: 250,000$ & & \\
\hline 2 & $d=d_{0}: 125,000$ & $d=d_{0} / \sqrt[3]{2} \approx 0.7937 d_{0}: 110,622$ & $d=\sqrt[3]{2} d_{0} \approx 1.260 d_{0}: 34,844$ \\
\hline 3 & $d=d_{0}: 125,000$ & $d=d_{0} / \sqrt[3]{3} \approx 0.6934 d_{0}: 153,547$ & $d=\sqrt[3]{3} d_{0} \approx 1.442 d_{0}: 24,605$ \\
\hline 4 & $d=d_{0}: 125,000$ & $d=d_{0} / \sqrt[3]{4} \approx 0.6300 d_{0}: 193,243$ & $d=\sqrt[3]{4} d_{0} \approx 1.587 d_{0}: 19,172$ \\
\hline
\end{tabular}

